\title{
Die Natur der Kongresse?
}

\section{Von Jochen Groß}

Nachdem die Soziologie mit dem Münchner Kongress der Deutschen Gesellschaft für Soziologie (DGS) 2004 Thomas Krons Einschätzung in der Kölner Zeitschrift für Soziologie und Sozialpsychologie zufolge aus dem »Jammertal « (2004, S. 786) geschritten ist, muss man sich bei Betrachtung des diesjährigen Kongresses in Kassel die Frage stellen, ob der »Patient Soziologie« (ebd.) weiter genesen konnte.

Thematisch konnten die lokalen Organisatoren Heinz Bude und Johannes Weiß unbestreitbar an das glücklich gewählte Münchner Thema (»Soziale Ungleichheit - kulturelle Unterschiede «) anknüpfen und mit der »Natur der Gesellschaft« ein gleichermaßen aktuelles, soziologisch breit zugängliches wie auch (potenziell) außenwirksames Thema bieten. In seinem Eröffnungsvortrag wies Karl-Siegbert Rehberg, Vorsitzender der DGS, ebenfalls auf die mit dem Thema verbundenen Chancen hin und skizzierte zugleich den »Kampf der Disziplinen« zwischen natur- und sozialwissenschaftlichen Perspektiven als wesentliches »Bedeutungsfeld « des Kongresses (Rehberg 2006).

Das Verhältnis von Natur- und Sozialwissenschaften wurde auf dem Kongress selbst hingegen wenig offensiv in Form der Präsentation eigenständiger soziologischer Ergebnisse angegangen. Vielmehr dominierten wissenschaftsreflexive Diskussionen, in denen die Dominanz der Naturwissenschaften beklagt wurde und man erstaunt deren Arroganz konstatierte, abschließende Erklärungen liefern zu wollen.

In ihrer Mittagsvorlesung positionierte sich Renate Mayntz zu dieser Diskussion am offensivsten. Die Preisträgerin der DGS für ein hervorragendes wissenschaftliches Lebenswerk und ehemalige Max-Planck-Direktorin sieht die zentrale Herausforderung der Soziologie durch die Naturwissenschaften darin, dass insbesondere »die moderne Genomik (...) das Verhältnis zwischen Natur und Umwelt, nature and nurture in der Bestimmung des menschlichen Handelns zugunsten der Natur zu verschieben« trachte (Mayntz 2006). In voller Konsequenz, so Mayntz, würde hier »die handlungstheoretische Basis der Soziologie« in Frage gestellt (ebd.). Ihr Vortrag war ein engagiertes Plädoyer für die Berechtigung, ja Notwendigkeit sowohl der Natur- als auch der Sozialwissenschaften. So sind letztere nicht ohne die Natur zu denken, denn sie prägt zweifelsohne jede Gesellschaft, doch der von der Soziologie betrachtete Mensch wird zurecht zuvorderst als »Produkt der Sozialisation in eine historisch geformte Gesellschaft« gesehen (ebd.). Dabei identifizierte Mayntz auch Befürchtungen, dass neurologische Erkenntnisse, wonach Menschen unbewusster handeln als die Soziologie es gemeinhin annimmt, als nur scheinbare Bedrohung. Zumindest dann, wenn wir uns als Disziplin auf ein gemeinsames Forschungsprogramm verständigen würden, das soziale Regelmäßigkeiten mittels sozialer Mechanismen auf Basis einfacher Handlungstheorien aufzudecken sucht. Insofern, so Mayntz, ist kein Widerspruch zwischen den Natur- und den Sozialwissenschaften zu erkennen, doch die Soziologie sollte sich auf ein brauchbares Theoriegerüst stützen und ihren Erklärungsanspruch nicht aufgeben, soll die scheinbare Bedrohung nicht doch real werden.

Über den wissenschaftlichen Anspruch der Soziologie und jenes von Mayntz vehement eingefordertes Forschungsprogramm machte man sich auf dem Kasseler Kongress viele Gedanken. Wieder einmal wurde deutlich, dass der Graben zwischen den konkurrierenden Wissenschaftsverständnissen und damit korrespondierend konfligierenden Theoriebegriffen tief wie eh und je ist. So beherzt man das Plädoyer von Mayntz aufgreifen und ihren Optimismus teilen möchte, erscheint die deutsche Soziologie kaum in der Lage, ein solches Forschungs-

Soziale Welt 58 (2007), S. 95 - 99 
programm auch konsequent umzusetzen. Dabei wäre eine Verständigung darüber, dass wir soziale Phänomene zum Beispiel entlang des Idealbilds des deduktiv-nomologischen Schemas erklären wollen, eine zentrale Voraussetzung für das Ausbrechen aus der »Dominanz der Lebenswissenschaften« wie sie Rehberg in seinem Eröffnungsvortrag beschrieb.

Die Ausrichtung an Hempel und Oppenheims (1948) grundlegenden Forderungen an vollständige Erklärungen kennzeichnet nicht nur die naturwissenschaftliche Forschung, sondern begünstigte zugleich deren Erfolg. Mayntz (2005) hat in einem kürzlich veröffentlichten Aufsatz den Erfolg der Naturwissenschaften zudem auf deren technische Fortschritte zurückgeführt, die immer genauere Beobachtungen und Messungen und somit einen kumulativen Wissensfortschritt ermöglichen. Die Sozialwissenschaften mit stetig wechselnden Themen und deren ebenso wechselnden Interpretation hingegen erwecken eher den Anschein einer additiven Wissenschaftsentwicklung. Man mag die stetige Reflexion der eigenen Wissenschaftlichkeit und Ziele als grundlegend und gewinnbringend betrachten. Dies sollte angesichts der anregenden Diskussionen, wie sie in der Sitzung der Sektion Soziologische Theorie anhand der Vorträge von Andrea Maurer und Andreas Pettenkofer über mechanismische Erklärungen oder auch in der Ad-hoc-Gruppe »Zur Ontologie der Badewanne - sozialtheoretische Probleme des Makro-Mikro-Makro-Erklärungsmodells« geführt wurden, auch unterstrichen werden. Jedoch führt diese anhaltende Diskussion nicht aus der Welt nebeneinander stehender und neu daneben gestellter Wissensbausteine hinaus. Allerdings zeigten sich auf diesem 33. Kongress der DGS auch Entwicklungen, die durchaus für den großen Einfluss technischer Neuerungen auf die Sozialwissenschaften sprechen - und verbunden mit theoriegeleiteter, methodisch reflektierter empirischer Sozialforschung durchaus den Weg des kumulativen Wissensgewinns weisen. Die präsentierten Befunde stützen sich auf immer elaboriertere Datenanalyseverfahren und auch die verfügbaren und dank rasanter computertechnischer Innovationen nutzbare Datenquellen erlauben immer detailliertere Blicke in gesellschaftliche Teilbereiche. Als Grundlage für solche Analysen dienen etwa die im Rahmen der Ad-hoc-Gruppe zu »Arbeitsmarktpartizipation, Erwerbsbiografien und sozialer Sicherung « von den öffentlichen Forschungsdatenzentren vorgestellten Datensätze der statistischen Ämter, der Bundesagentur für Arbeit und auch der Rentenversicherungsträger.

Ebenfalls ermutigend erscheinen die präsentierten Beiträge, die weniger aus einer wissenschaftstheoretischen Perspektive heraus Akzente setzten, sondern auf Grundlage von Handlungstheorien soziale Prozesse erklärend in Blick nahmen und anhand empirischem Material prüften. Diese fanden sich in einigen angebotenen Veranstaltungen wie der Sitzung der Sektion Modellbildung und Simulation und einigen Ad-hoc-Gruppen. Verbindungsfähig mit den Naturwissenschaften erscheinen im Übrigen netzwerkanalytische Arbeiten wie sie im Plenum »Netzwerk-Gesellschaft« oder auch der daran anknüpfenden Ad-hoc-Gruppe »Netzwerkanalyse« vorgestellt wurden.

Einen weiteren zentralen Aspekt der Auseinandersetzung von Natur- und Sozialwissenschaften hätte man im Rahmen der hochschulpolitischen Diskussionen, die über die Foren und Abendveranstaltungen weit in das »reguläre « Programm hineinreichten, angesichts der offenkundigen Nachrangigkeit der Sozialwissenschaften bei der Drittmittelvergabe erwarten können. ${ }^{1}$ Hier drängte sich jedoch der Eindruck auf, dass weniger die Naturwissenschaften, sondern mehr die Ökonomik die Soziologie fordert. In der Plenarveranstaltung »Schicksal Markt - Ökonomisierung als >Survival of the Fittest«« nahm Uwe Schimank die Ökonomisierung der Hochschulen in Blick. Einleuchtend beschrieb er das Eindringen ökonomischer Handlungslogiken in die Hochschullandschaft - oder allgemeiner in nicht-wirtschaftliche In-

1) Die Entscheidung über die Vergabe der Exzellenzcluster der Deutschen Forschungsgemeinschaft und des Wissenschaftsrates wurde am letzten Kongresstag bekannt gegeben. 
stitutionen - und forderte eine Erklärung dieses Phänomens. Schimank beschrieb wesentliche Steuerungsmechanismen im Hochschulsystem und identifizierte international einen Governancewechsel vom traditionellen Regime zu New Public Management-Strukturen, die insbesondere durch die Mechanismen »Konkurrenzdruck« und »Hierarchische Selbststeuerung der Hochschule« gekennzeichnet sind. Allerdings konnte Schimank zu diesem Zeitpunkt des Forschungsprojekts noch keine befriedigende Erklärung der Ausbreitung ökonomischer Handlungsweisen liefern. Nun sollten wir hier die Hoffnung hegen, dass die erfolgte Deskription des Geschehenen und die Identifikation möglicher dahinter stehender Mechanismen ein erster Schritt zur Erklärung ist. Aus den in Bezug auf die Ökonomisierung von Hochschulen weit fortgeschrittenen Entwicklungen in Australien und Großbritannien folgerte Schimank für Deutschland einen ähnlichen Verlauf und zeigte dessen Konsequenzen auf: Wissenschaftler als Auftragsprofis, Kurzfristopportunismus und die Entkopplung von Forschung und Lehre. Auch wenn die Ursachen für diese Prozesse noch ungeklärt erscheinen, fordern diese, sich auch hierzulande schon abzeichnenden Konsequenzen, heraus.

Diese wurden im Forum »Hochschulpolitik zwischen Leitungsautoritarismus und PersonalVerschrottung « aufgenommen. Das Forum richtete sich explizit normativ gegen das von Schimank als Explanandum definierte soziale Phänomen der Ökonomisierung der Hochschulen. Vor dem Hintergrund der zahlreichen hochschulpolitischen Änderungsprozesse (Elite-Universitäten, Exzellenzcluster, Bachelor- und Masterstudiengänge etc.) sollten hier »aktuelle Tendenzen der Hochschulgesetzgebung « (Ankündigung im Programmheft) diskutiert werden. Schade nur, dass die Diskutanten, Reinhard Blommert, Paul Kellermann, Elisabeth Meyer-Renschhausen sowie Heinz Steinert, aufgrund ihrer übereinstimmenden Ideologiekritik am »Diktat der so genannten Lissabon-Strategie der Europäischen Union« keine Diskussionsgrundlage hatten und der Zuhörerschaft lange Einzelstatements über die Universitätsreformen als »Fortsetzung der volkswirtschaftlichen Wertschöpfungskette«, begründet durch die »US-neokonservative Politik der Privatisierung « (Blomert) in verschwörungstheoretischer Manier feilboten. Diese »Diskussion « erschien insbesondere deshalb müßig, weil es versäumt wurde, die empirisch festzustellenden Paradoxien klar zu benennen, die etwa im Zusammenhang mit dem Bologna-Prozesses von Rehberg in seiner Forumseinführung verdeutlicht wurden. Rehberg konstatierte, dass bei der Vereinheitlichung der Studienbedingungen durch die Einführung konsekutiver Studiengänge paradoxerweise in Bezug auf die intendierte Internationalisierung schon jetzt ein gegenteiliger Effekt zu beobachten sei und die Internationalität so gefährdet sei wie nie. Es ließen sich weitaus mehr und ebenso schwer wiegende Kritikpunkte an der deutschen Umsetzung des Bologna-Programms ausmachen, die man ansprechen, kritisieren und auch politisch zu verändern suchen sollte. Doch leider trugen die Diskutanten plumpe Ideologiekritik vor, die den Washington Consensus bemühte, anstatt konstruktiv und differenziert argumentierend gegen die praktischen Umsetzungsprobleme vor Ort und nichtintendierte Folgen der Reformen anzugehen. So wurden unbestreitbare positive Absichten und auch bereits zu beobachtende Effekte - wie sie etwa von Josef Brüderl formuliert wurden - in Gänze ignoriert und in Abrede gestellt. Zu denken ist hier beispielsweise an die sinkenden Abbruchquoten, die insgesamt kürzere Zeit bis zur Erreichung eines akademischen Abschlusses und auch die umfassenden Möglichkeiten der Neugestaltung teilweise stark angestaubter Curricula. Agiert man derart, darf man sich nicht über die mangelnde Öffentlichkeitswirksamkeit des Faches wundern. Insofern kann man Jürgen Kaubes Charakterisierung des Kongressgeschehens in der Frankfurter Allgemeinen Zeitung »als völlig diskussionslos gebliebenes Abfeiern des Unzusammenhängenden« (Kaube 2006) gut nachvollziehen.

Als beobachtender Kongressbesucher bleibt insbesondere festzuhalten, dass immer nur ein subjektiv ausgewählter Ausschnitt des Angebots wahrgenommen werden kann. So fiel etwa die gesamte - sicher interessante - Veranstaltungsvielzahl zum Gastland China anderen Programmpunkten zum Opfer. Dieser Umstand der Erfahrung nur einer vergleichsweise gerin- 
gen Zahl an Vorträgen scheint wesentlich die »Natur « eines solchen Großkongresses zu bestimmen - ebenso wie die auf der Heimreise gemischte Einschätzung des Gehörten und Gesehenen. Einerseits ärgerte man sich über den Zuhörer ratlos lassende Veranstaltungen wie die erwähnte Forumsveranstaltung oder die begrüßenswerte, jedoch überfüllte und an einem akustisch ungünstigen Ort abgehaltene Nachwuchsveranstaltung »Hochschulkarriere als Hazard «. Andererseits erinnert man sich gerne an die persönlich erlebten Glanzlichter wissenschaftlicher Betätigung. So berichteten Paul Windolf und Lothar Krempel nachhaltig eindrucksvoll unter Rückgriff auf netzwerkanalytische Verfahren über die Entwicklungen der Unternehmensverflechtungen in Deutschland (Plenum »Netzwerk-Gesellschaft«) und Andrea Maurer hat mit ihrem Sektionssitzungsvortrag wie oben bereits erwähnt die Diskussion über Erklärungen in den Sozialwissenschaften verdienstvoll angeregt (vgl. dazu auch Hedström/Swedberg 1998; Schmid 2006).

Daneben sind sicher die zahlreichen kleinen Begebenheiten eines solchen Zusammentreffens der deutschen Soziologie hervorzuheben. So eignet sich eine solche Veranstaltung immer, um neue Kontakte zu knüpfen und um Namen mit Gesichtern zu verbinden. Gleichwohl muss man aus inhaltlicher Perspektive sehen, dass in kleinem Rahmen angelegte Fachkongresse wie beispielsweise Sektionstagungen tiefere und mitunter auch neuere Einblicke in das eigene Forschungsfeld bieten. Diese waren in Kassel entsprechend eher im Rahmen des dicht gepackten Nachmittagsprogramms zu finden und nicht in den großen Plenarsitzungen. Seitens der Organisatoren hätte man sich eine striktere Begrenzung der Zahl der Ad-hocGruppen gewünscht sowie eine bessere Verteilung der Veranstaltungen auf den ganzen Tag gerne auch zu Ungunsten der eher exklusiv gehaltenen Plenen. Dennoch ermöglicht der zweijährliche DGS-Kongress - und das ist denke ich zweifellos eine Stärke - Einblick in die gesamte Breite der Forschungsanstrengungen der Disziplin. An dieser Stelle soll nicht verschwiegen werden, dass mancher dieser abschweifenden Blicke über die eigenen üblichen Beschäftigungsfelder hinaus auch organisatorischen Zwängen anzulasten ist. Offenkundige Schnittmengen von Veranstaltungen wie bei den Sitzungen der Methoden- und der Modellbildungssektionen und auch einer Ad-hoc-Gruppe, die an das Netzwerkplenum letzt genannter Sektion anschloss, wurden seitens der Programmgestalter nicht berücksichtigt. Dies zwang entweder zum ausgiebigen »Veranstaltungshopping « oder bedingte die Partizipation an als unattraktiver empfundenen Programmpunkten. Der teilweise »erzwungene« Blick auf das gesamte Fach mag durchaus manche Zweifel an der Disziplin begründen, doch dieses scheinbare »Abfeiern des Unzusammenhängenden« (Kaube 2006) zeigte auch den Reiz der Soziologie. Hier reflektiert und streitet man noch über die eigenen wissenschaftlichen Ansprüche und stellt Differenzen in den Vordergrund.

Lernen an anderen Disziplinen ist dennoch angeraten, insbesondere bei einem Blick auf die Professionalisierung. Dazu ist sicher die Etablierung einer neuen Vortragskultur überfällig. So sollten die Veranstalter das Ablesen ausgearbeiteter Manuskripte ebenso aus dem Kongressalltag verbannen wie ein angemessenes Zeitmanagement durchsetzen. Der DGSKongress bzw. zumindest ein Großteil der Veranstaltungen gewinnen ihren Charakter und die Lernchancen durch die Diskussion. Ermöglicht man jedem Vortragenden / Diskutanten eine Redezeit bis zur Ausfüllung der Veranstaltungszeit, wird dieses wesentliche Ziel verfehlt und es stellt sich eine zumindest latente Frustration bei den Teilnehmern und - so der Eindruck - auch bei den Vortragenden ein.

Zur Professionalisierung gehört auch die von Jutta Allmendinger schon 2000 geforderte und auch eingeführte Öffnung der DGS gegenüber Studierenden. Auch der Kasseler Kongress bot diesbezüglich eine erfreuliche Neuigkeit. Das erstmals angebotene Fachschaftsticket zog eine große Zahl von Studierenden an, die nur als Bereicherung des Kongresses gewertet werden können. Diese konsequente Heranführung von Studierenden an die Forschungsarbeiten des Fachs gehört ebenso wie der permanente Blick auf die Förderung 
des wissenschaftlichen Nachwuchses zu zwingenden Elementen der Professionalisierung. Zunehmend bedeutungsvoll dürfte vor dem Hintergrund der Etablierung von Bachelor- und Masterstudiengängen die Pflege der Kontakte in die außeruniversitäre Berufspraxis werden. Auch diesbezüglich scheint man in der Soziologie auf einem richtigen Weg und organisierte eine spezielle Veranstaltung (die im Übrigen sehr gut besucht war) zu »Schlüsselqualifikationen von Soziologen«.

Schließlich ist als weiterer zentraler Aspekt der Professionalisierung die Öffentlichkeitsarbeit zu thematisieren. Die Vielfältigkeit der Angebote belebt zwar für den Fachbesucher den Wert eines solchen Kongresses, jedoch erkauft man sich dadurch die Gefahr der Beliebigkeit. Mit der »Natur der Gesellschaft« und den von Rehberg damit assoziierten »Bedeutungsfeldern « befasste sich wohl nur eine Minderzahl der Veranstaltungen - ein Grund womöglich, den Kongress als »Inklusionsparty« (Kaube 2006) zu beschreiben. Dies erscheint keineswegs falsch, denn auch der diesjährige Kongress war vorrangig ein Schaulaufen von professionellen Akteuren. Diese Unbestimmtheit trägt sicher nicht zu einer wirksameren Wahrnehmung der Soziologie in der Öffentlichkeit und der Professionalisierung der Außenkommunikation bei, zumal offenkundig wurde, dass es der Soziologie nicht möglich erscheint, sich gemeinsam mit einem - noch so grundlegenden - Themenkomplex auseinanderzusetzen. Mir erscheint dies zwar nicht als zentraler Angriffspunkt gegen die Soziologie oder den Nutzen des Kongresses, doch die wieder hervorgetretene Deutlichkeit des Unmöglichen ein einheitliches Wissenschaftsverständnis geschweige denn Forschungsprogramm zu entwickeln, das auf kumulativen Wissensfortschritt abzielt, betrübt die Gesamteinschätzung. Insofern ist mit der von Kron (2004) aufgrund der positiven Bewertungen des Münchner Kongresses erhofften Genesung des »Patienten Soziologie« (Kron 2004) vermutlich auch beim nächsten Mal 2008 in Jena nicht zu rechnen.

\section{Literatur:}

Hedström, Peter / Swedberg, Richard (1998): Social Mechanisms: An Introductionary Essay, in: Dies. (Hrsg.): Social Mechanisms. An Analytical Approach to Social Theory. Cambridge, S. 1-31.

Hempel, Carl G. / Oppenheim, Paul (1948): Studies in the Logic of Explanation, in: Philosophy of Science 15, S. 135-175.

Kaube, Jürgen (2006): Naturbulent. Inklusionsparty: Der Soziologentag in Kassel, in: Frankfurter Allgemeine Zeitung 241, S. 39.

Kron, Thomas (2004): Die Außenwirkung der Innenwirkung - die Soziologie schreitet aus dem Jammertal, in: Kölner Zeitschrift für Soziologie und Sozialpsychologie 56, S. 786-787.

Mayntz, Renate (2005): Forschungsmethoden und Erkenntnispotential. Natur- und Sozialwissenschaften im Vergleich. MPIfG Discussion Paper 05/7, Köln.

Mayntz, Renate (2006): Einladung zum Schattenboxen: Die Soziologie und die moderne Biologie, in: Abstractband zum 33. Kongress der Deutschen Gesellschaft für Soziologie in Kassel, S. 393.

Rehberg, Karl-Siegbert (2006): Soziologie als »Wirklichkeitswissenschaft« jenseits von Naturalismus und Virtualitätseuphorie. Eröffnungsvortrag des 33. Kongresses der Deutschen Gesellschaft für Soziologie. Unveröffentlichtes Redemanuskript, Dresden.

Schmid, Michael (2006): Die Logik mechanismischer Erklärungen, Wiesbaden.

Jochen Groß, M.A.

Institut für Soziologie

LMU München

Konradstr. 6

80801 München

e-mail: jochen.gross@soziologie.uni-muenchen.de 